\title{
Wiener's Construction of the Brownian Motion
}

\subsection{Preliminary Remark: The Space of All Paths}

Illustration of the Brownian Motion in Textbooks In many economic textbooks which address the Brownian motion one finds representations resembling those of Fig. 6.1. Let us initially focus on the blue path, a function frequently used to illustrate a typical path of a Brownian motion. Almost everyone would accept that the price of a share could develop as shown. In particular, economists find such a representation plausible. However, such an interpretation is more likely to mislead rather than to contribute to the understanding of what the Brownian motion is all about. Even worse, they convey a misconception of the Brownian motion. Let us explain this phenomenon by looking at a coin toss.

With a coin toss we have always assumed that the only events possible are heads and tails. Of course certain other events are conceivable: one possibility is that a coin falls on its edge as shown in Fig. 6.2, or the coin could disintegrate in several pieces or it could entirely disappear. Yet all these possibilities are highly unlikely.

Does Fig. 6.2 describe the random outcome of the coin toss adequately? Certainly not! In fact, the picture can be called misleading. The same argumentation also applies to the blue path shown in Fig. 6.1! This single path represents only one of an infinite number of possible paths. Rather, the Brownian motion must be considered as a dice with an infinite number of sides (instead six) where the outcome is a continuous function. Any function is called a path or an event; and every single path chosen is just as unlikely as the event shown in Fig. 6.2.

Returning to Fig. 6.1 let us concentrate on the black path. With respect to stock prices everybody would consider the black path as an unlikely path because of its untypical (sinusoidale) shape: but the shape does not matter. Thus the blue path is as unlikely as the black path because both of them represent just one of an infinite number of continuous functions. 


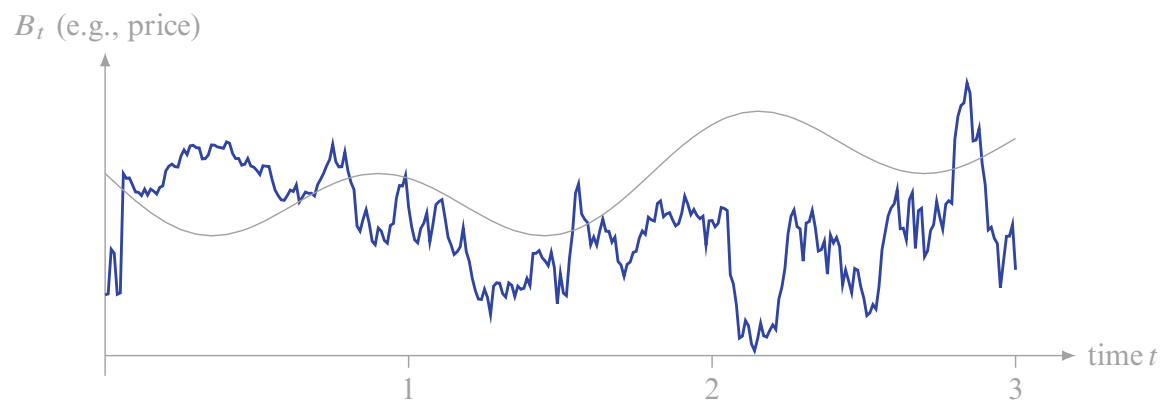

Fig. 6.1 Textbook description of a Brownian motion

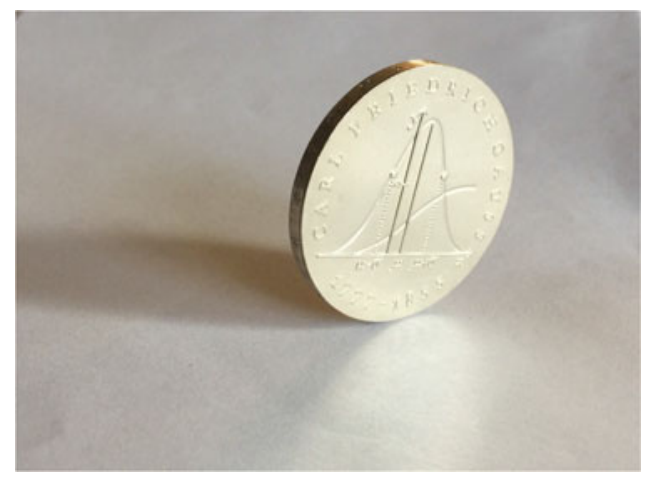

Fig. 6.2 Unlikely result of a coin toss (this East German coin was issued to commemorate the 200th anniversary of Gauss' birthday displaying the normal distribution)

If Fig. 6.2 as a typical coin toss triggers discomfort, why doesn't Fig. 6.1 cause similar discomfort when it comes to Brownian motion?

From Single Numbers to Intervals Concentrate on a situation in which the random development of a company's cash flows over time is of concern. Managers responsible for planning may assume that the cash flows in the coming year will be equally distributed in the interval between $\$ 3$ million and $\$ 4$ million. A decisionmaker could be interested in the consequences of the cash flows being exactly $\$ \pi$ million. ${ }^{1}$ A reasonable person would dismiss such a discussion as a purely academic gimmick: with an evenly distributed random variable the probability of a specific real number is zero. This case does not have to be discussed any further since it is absolutely unlikely. It makes much more sense to select a relevant interval for the cash flows, for example, between $\$ 3.00$ million and $\$ 3.25$ million and to study their effect on important business parameters such as profit, investment volume, or firm value. Subsequently, the analysis of other cash flow intervals is

\footnotetext{
${ }^{1}$ That would be $\$ 3,141,592$ if we limit ourselves to full dollar amounts.
} 
recommended. Only in this way one gets a useful understanding of the economic consequences resulting from the assumption of equally distributed cash flows between $\$ 3$ million and \$4 million.

From a Single Path to All Paths After these reflections we return to Fig. 6.1 showing two paths of a Brownian motion. As the probability of a payment of $\$ \pi$ million is zero, the same applies to the probability of each of the two paths. In fact, it is quite conceivable that the cash flows will follow not the sinusoidal but the blue movement that seems to be random. From the perspective of probability theory, both the sinusoidal and the blue movement are unlikely. In order to arrive at meaningful statements, we must focus on the entire event space of the Brownian motion. Looking at individual paths is meaningless. Rather, one must consider all paths.

From Universal Statements to Probability Statements Unfortunately, by simply looking at all paths $C[0, \infty)$ one goes too far. Stating "A particular property $x y z$ applies to all paths of the set $C[0, \infty)$ " could cause a problem. By doing so we would include paths that are not of interest because nobody would consider them as random (remember the sinusoidal path!). While these functions are annoying, they still do exist. A "trick" is required to at least ignore or disregard them.

The crucial step in disregarding annoying functions is to switch from universal to probability statements. ${ }^{2}$ Unfortunately, this switch demands a hard-to-read double negation. We have to state: "It is not likely that the property $x y z$ does not apply to all paths." This is the trick allowing us to ignore the annoying functions. The same result, however, can also be achieved by the following (positive) statement: a set of paths has the property $x y z$ almost everywhere, if and only if the set's probability equals 1 .

Then the only question remaining is how to construct the relevant probability. This probability is the so-called Wiener measure in the space of continuous functions. In the preceding chapters we have developed important foundations ( $\sigma$ algebra, definition of the measure) for defining this measure. Once the Wiener measure $\mu$ is defined we can state the following: a Brownian motion "has property $x y z$," if and only if the set of functions $C[0, \infty)$ with this property has measure 1 . Table 6.1 illustrates our remarks.

Let us summarize. The definition of the Brownian motion we will present shortly may irritate readers with economic backgrounds. Why?

1. Economists tend to look at Brownian motion by considering only a few paths, perhaps two, ten, or a hundred, instead of recognizing that this stochastic process consists of an infinite number of paths. This characteristic of the Brownian motion is easily overlooked as the aspect of infinity manifests itself only in the inconspicuous symbol $C[0, \infty)$.

\footnotetext{
${ }^{2}$ We had seen in Definition 3.3 on page 55 how one can "get rid of" annoying properties of an object with the term almost everywhere.
} 
Table 6.1 Analogy between coin toss and Brownian motion

\begin{tabular}{l|l|l}
\hline & Ideal coin & Brownian motion \\
\hline State & Coin face & Path \\
\hline Event space & Heads or tails & $\begin{array}{l}\text { All continuous functions } \\
{[0, \infty) \rightarrow \mathbb{R}}\end{array}$ \\
\hline Probability & $\frac{1}{2}$ & Wiener measure $\mu$ \\
\hline
\end{tabular}

2. In addition to considering only few paths, many economists attach importance to the scope of individual paths. The focus of the definition on page 93, however, will be placed on something quite different: the focus has to be the probability $\mu$ which is assigned to the sets of paths. This is of crucial importance. Some economists may not even realize that such a probability exists.

Two further remarks seem to be necessary. First, individual paths of the Brownian motion cannot be differentiated at almost any point. Second, almost all paths are non-monotonous in any interval as small as the interval may be. Economists not having a sufficient mathematical background may fail to appreciate the significance of those statements. ${ }^{3}$ Without detailing these mathematical properties we can only state that the paths can by no means look as shown in Fig. 6.1. Accounting for these two remarks the typical paths or jagged functions frequently found in economic textbooks are anything but typical.

\subsection{Wiener Measure on the Space of Continuous Functions}

Equipped with the background presented in the previous chapters we are approaching the core of our book. The following material is based on the American mathematician Norbert Wiener who in the 1920s put the Brownian motion on solid mathematical grounds.

Binomial Model and Space of Continuous Functions We had already illustrated a binomial model on page 25 in Fig. 2.5. A path is a complete "one-way tour" through a tree from its origin to one of the ends.

Contrary to the binomial model the paths in a Brownian motion are not based on sequential upward or downward movements of an economic quantity. Rather, a path is a continuous function. Furthermore, no additional assumptions are needed, in particular the functions do neither have to be differentiable nor monotonous. Each of these continuous functions describes how the relevant variable can develop in time.

\footnotetext{
${ }^{3}$ We will give corresponding explanations on page 95 .
} 


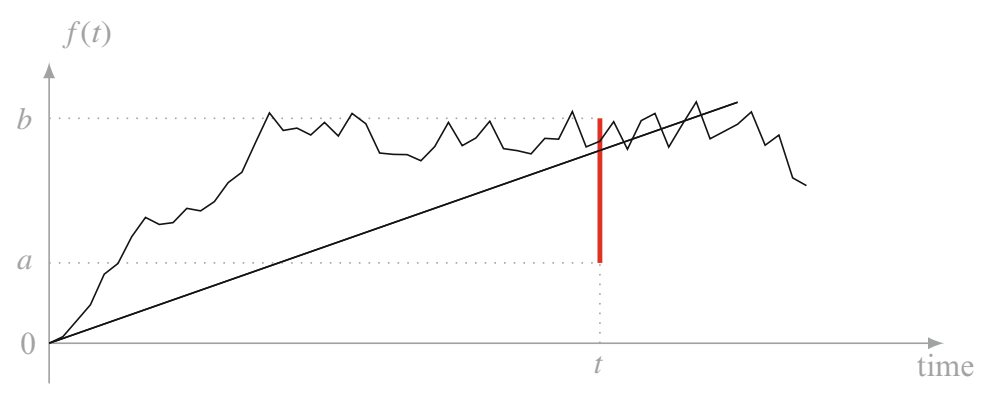

Fig. 6.3 Two elementary events in the event space $C[0, \infty)$ from Fig. 3.5

The Measure of a Set of Continuous Functions In order to determine the measure of a set of continuous functions we must first clarify which sets can be measured at all. This task was described in our Example 3.7 on pages 48 to page 50. We are following the pattern used to construct the $\sigma$-algebra. The scheme consisted of a finite number of design steps. ${ }^{4}$ In the first step, an arbitrary time $t$ is selected and an interval $[a, b]$ is chosen. Thus, a measurable set contains all functions passing through the interval $[a, b]$ at time $t$.

Let us look at of all these functions. For this purpose we redraw Fig. 3.5 from page 48 but eliminate the sinusoidal path as it does not go through the $[a, b]$ interval at time $t$; therefore, it does not belong to the set to be measured (Fig. 6.3). Remember, that the set of all paths going through the $[a, b]$ interval is called a cylinder set.

We determine the measure of this cylinder set as follows ${ }^{5}$ :

$$
\mu(\{f: f(t) \in[a, b]\}):=\int_{a}^{b} \phi_{t}(x) d x .
$$

It is easy to see that the measure depends on both time $t$ and the interval $[a, b]$. With increasing time $t$ the measure of the cylinder set decreases because the smaller the density, the larger the variance. And the larger the interval $[a, b]$, the larger is the measure of this set.

The largest possible value of a measure of any cylinder set is 1 implying that the set contains all continuous functions. ${ }^{6}$ Since the density function is never negative the Wiener measure is a probability measure.

\footnotetext{
${ }^{4}$ For details refer to pages 48 to 50 .

${ }^{5}$ Here $\phi_{\sigma^{2}}(\cdot)$ represents the density of the normal distribution with expectation 0 and variance $\sigma^{2}$. ${ }^{6}$ This is the borderline case for $a \rightarrow-\infty$ and $b \rightarrow \infty$ for any time $t$.
} 
Usually one denotes the antiderivative of the density function $\phi_{t}(x)$, i.e., the distribution function, with the symbol $\Phi_{t}(x)$. Using the fundamental theorem of calculus we can write the Wiener measure in the following form:

$$
\mu(\{f: f(t) \in[a, b]\})=\Phi_{t}(b)-\Phi_{t}(a) .
$$

Let us determine the measure if the length of interval $[a, b]$ goes to zero. In the case of $a=b$, the measure is obviously zero. But for an infinitesimal small interval $[x, x+d x]$ the difference of the distribution functions $\Phi_{t}(x+d x)-\Phi_{t}(x)$ tends to $\phi_{t}(x) d x$. The resulting measure is

$$
\mu(\{f: f(t) \in[x, x+d x]\})=\phi_{t}(x) d x .
$$

We will use this equation on page 95.

In the next step we define the Wiener measure of a cylinder set having not only one but two points in time. Remember the construction rules on page 49 (Fig. 3.6): the paths belonging to this cylinder set have the property that they traverse certain intervals. At the first (earlier) time $t$ the function value $f(t)$ must lie in the interval $[a, b]$; at the second (later) time $s$ the difference of the function values $f(s)-f(t)$ must run through the interval $[c, d]$. The measure is defined as follows:

$$
\begin{gathered}
\mu(\{f: f(t) \in[a, b] \text { and } f(s)-f(t) \in[c, d]\}) \\
\quad=\int_{a}^{b} \int_{c}^{d} \phi_{t}(x) \phi_{s-t}(y-x) d x d y .
\end{gathered}
$$

This definition requires further explanation. We see two integrals. First, we recognize the integral over $x$ contained in the interval $[a, b]$ which we already used above. Second, we can identify another integral over a variable $y$ contained in the interval $[c, d]$. The difference $y-x$ is normally distributed with variance $s-t$.

The definition is easier to understand if one looks at small intervals $[x, x+d x]$ and $[y, y+d y]$ instead of the (arbitrarily large) intervals $[a, b]$ and $[c, d]$. Using a notation similar to (6.3) with density functions leads to

$$
\begin{aligned}
& \mu(\{f: f(t) \in[x, x+d x] \text { and } f(s)-f(t) \in[y, y+d y]\}) \\
& \quad=\phi_{t}(x) \phi_{s-t}(y) d x d y .
\end{aligned}
$$

Equation (6.5) highlights that the product of two density functions must be determined in order to obtain the measure of an infinitesimal small range. The product of density functions comes into play with independent quantities. Thus, we can see that the function value $f(t)$ at time $t$ should be independent of its further development described by the difference $f(s)-f(t)$.

For each further point in time multiply expression (6.5) with the additional term $\phi_{t_{n+1}-t_{n}}(\cdot)$. Again the variance of this normal distribution depends on its distance to 
the earlier points in time. For example, the measure for the cylinder set considering a third time $r>s$ results in

$$
\begin{aligned}
& \mu(\{f: f(t) \in[x, x+d x] \text { and } f(s)-f(t) \in[y, y+d y] \\
& \quad \text { and } f(r)-f(s) \in[z, z+d z]\})=\phi_{t}(x) \phi_{s-t}(y) \phi_{r-s}(z) d x d y d z .
\end{aligned}
$$

\subsection{Two Definitions of the Brownian Motion}

At last, we can define the Brownian motion formally.

Definition 6.1 (Brownian Motion, Mathematically) A Brownian motion is given by a probability space $(C[0, \infty), \sigma$-Algebra, $\mu)$ with $\mu$ as Wiener measure. ${ }^{7}$

This definition is extraordinarily terse and cannot be beaten in brevity. The definition uses terms which are not easily understood by non-mathematicians. However, we hope that careful reading of the previous chapters of this book will help to overcome any obstacles.

Economists usually define the Brownian motion quite differently. ${ }^{8}$

Definition 6.2 (Brownian Motion, Economically) The Brownian motion $W(t)$ meets the following three properties:

1. Starting at the origin: $W(0)=0$ applies.

2. Continuity: $W(t)$ is continuous in $t$.

3. Distribution: the increments $W(s)-W(t)$ for $s>t$ are normally distributed with expectation zero and variance $s-t$. For adjacent time intervals the increments are independent of each other.

One might be inclined to think that both definitions express very different objects. However, that is only seemingly so. In fact, both definitions are equivalent!

Equivalence of Both Definitions It is possible to prove that Definition 6.2 can be derived from Definition 6.1. To realize this, we first need to understand the meaning of $W(t)$.

$W(t)$ is a random variable. More precisely, the random variable $W(t)$ returns a real number for every event. This real number depends not only on time $t$ but also on the event. In Chap. 4 (on page 59) we have stated that these events are the cause for the observed result. What are causes and events in this definition? Further,

\footnotetext{
${ }^{7}$ The associated $\sigma$-algebra was discussed on page 49. For the Wiener measure we refer to pages 91 to 93 .

${ }^{8}$ See for example Hassler (2007, p. 117).
} 
how can one imagine the functional dependency between individual events and the corresponding real numbers?

In Chap. 3 (on page 26) we had identified the set of all future events as space $\Omega=C[0, \infty)$. Each continuous function $f$ defined on the interval $[0, \infty)$ represents a possible event, in fact an elementary event. This event, i.e., this function $f$, determines the observed real number. While in the frequently used dice example only six possible elementary events are possible, in the Brownian motion an infinite number of elementary events do exist. The number of conceivable continuous functions $f$ on the interval $[0, \infty)$ cannot be counted. Any continuous function $f(\cdot)$ that can be drawn from the "C$C[0, \infty)$-lottery" represents a random event; and each of these functions returns a certain function value $f(t)$ at time $t$.

Thus, the random variable $W(t)$ can be described as follows. $W(t)$ is the random variable that assigns to an elementary event $f$, i.e., a continuous function, the value that this function $f$ assumes at time $t$. In order to describe $W(t)$ formally one can state

$$
W(t)(f \in C[0, \infty)):=f(t)
$$

random variable (event $f$ ) $:=$ numerical value.

In interpreting (6.7) one has to be careful because $f$ appears on both the left and the right, however, with two different meanings. On the left we see the notation of the random variable $W(t)$. The value of this random variable depends (causally) on a random event $f$ and such an event must be a function from the event space $C[0, \infty)$. On the right $f(t)$ is the value the randomly selected function $f$ assumes at time $t$.

The function $f$ performs two tasks in Eq. (6.7). Appearing on the left side of (6.7) it is the cause of uncertainty; it triggers the fluctuations of the Brownian motion. On the right side the function $f$ describes the fluctuation in detail by specifying for each $t$ how large the fluctuation will be. This is accomplished by the term $f(t)$.

To this point we have dealt with $W(t)$. We must now focus on the difference $W(s)-W(t)$ appearing in the economic definition presented above. This difference can be interpreted as a random variable and must therefore depend on a random event. This event must again be a continuous function $f \in C[0, \infty)$. Analog to the above considerations the result of the random variable is not the value of this function at time $t$ but its change between the times $t$ and $s$

$$
\begin{aligned}
(W(s)-W(t))(f \in C[0, \infty)) & :=f(s)-f(t) \\
\text { random variable (event) }) & :=\text { numerical value. }
\end{aligned}
$$

At last we can dedicate ourselves to prove that the mathematical Definition 6.1 actually fulfills the Properties 1 to 3 in the economic Definition 6.2.

Proof We will show that the three properties are true. 
1. Start at the origin: The random variable $W(0)$ always corresponds to the function value at $t=0$ for every conceivable event. Since all functions from the set $C[0, \infty)$ start by definition in zero, ${ }^{9}$ property 1 is satisfied.

2. Continuity: Continuity requires that each realization of the random variable (i.e., each event) represents a continuous function in $t$. Since the space $\Omega$ of the Wiener measure consists only of functions being continuous in $t$, property 2 is also satisfied.

3. Distribution: To prove that the increments of Brownian motion are independent and normally distributed, we can determine the distribution function. For this purpose we calculate the probability

$$
\operatorname{Prob}\{f:(W(s)-W(t))(f) \leq a\}=\mu(\{f:(W(s)-W(t))(f) \leq a\}) .
$$

We show that this expression corresponds to the normal distribution $\Phi_{s-t}$. Concentrating on Eq. (6.5) which defines the Wiener measure $\mu$, we immediately see the following two properties:

(a) The two density functions are multiplied by each other. This is to say that the random variables $W(t)$ and $W(s)-W(t)$ must be independent of each other.

(b) Both random variables are normally distributed with densities $\phi_{t}$ and $\phi_{s-t}$. This was to be shown.

\subsection{Often Neglected Properties of the Brownian Motion}

The following characteristics of the Brownian motion are often neglected in economic textbooks. Our purpose for discussing these properties is not for the sake of completeness. Rather, the understanding of further properties will enhance the skepticism in the use of Brownian motion when modeling economic processes.

Non-differentiability One can prove that the paths of Brownian motion cannot be differentiated $\mu$-almost everywhere. ${ }^{10}$

Figure 2.6 (page 27) illustrated that the sine function as well as the linear function represent conceivable paths of Brownian motion. Such functions are known to be differentiable. However, the probability that such paths will occur is zero. All of them are extremely unlikely.

While we do not intend to prove non-differentiability we at least want to make it plausible. For this purpose we concentrate on an arbitrary path $W(t)$ of the Brownian motion. Assuming that this path is differentiable implies that its derivative $W^{\prime}(t)$ exists.

\footnotetext{
${ }^{9}$ See page 27 .

${ }^{10}$ The phrase "almost everywhere" is described in Sect. 3.8.
} 
For differentiable functions the mean value theorem of differential calculus applies. This proposition says: if a function $f$ is differentiable on the closed interval $[a, b]$ (with $a<b$ ) and continuous on the open interval $(a, b)$, there exists at least one $s \in(a, b)$ with

$$
f^{\prime}(s)=\frac{f(b)-f(a)}{b-a} .
$$

For a random number $\varepsilon$ with an expected value of $\mathrm{E}[\varepsilon]=0$, it follows that there is a $s \in(t, t+\varepsilon)$ such that the difference between $W(t+\varepsilon)-W(t)$ can be estimated by

$$
W(t+\varepsilon)-W(t)=W^{\prime}(s) \cdot \varepsilon .
$$

This is the key to understanding the assertion that the paths of the Brownian motion cannot be differentiated. Equation (6.11) is an identity of two random variables implying that we can form variances on both sides. It follows from the properties of the Brownian motion that the left side of this equation is a normally distributed random variable with expectation zero and variance $t+\varepsilon-t=\varepsilon$. Thus

$$
\varepsilon=\operatorname{Var}[W(t+\varepsilon)-W(t)] .
$$

Further, the mean value theorem (6.11) tells us what happens on the right side,

$$
\operatorname{Var}\left[W^{\prime}(s) \cdot \varepsilon\right]=\varepsilon^{2} \operatorname{Var}\left[W^{\prime}(s)\right] .
$$

Combining the two equations results in

$$
\frac{1}{\varepsilon}=\operatorname{Var}\left[W^{\prime}(s)\right] .
$$

Letting $\varepsilon \rightarrow 0$ the left side of this equation approaches infinity. The right side of the equation goes to $W^{\prime}(t)$ and we have the logical contradiction $W^{\prime}(t)=\infty$.

Since the first derivative does not exist, the paths of a Brownian motion are $(\mu$ almost everywhere) not differentiable. Non-mathematical readers will most likely have trouble imagining such functions. We will hardly be able to change that.

Infinite Zero Crossings at the Beginning Many economist do not seem to care whether a path of a Brownian motion is differentiable or not. Further, we will discuss a property which is even more outrageous. We are talking about the intersections of any path with the abscissa: how often does such a path cross the abscissa in a certain time interval?

Again, we must realize that this is not about the behavior of a single path. Mind one should remember that the set of all paths of the Brownian motion must be considered. Thus, the only meaningful question is: what is the probability that the 
Fig. 6.4 Curve of the arccos function in the interval $[0,1]$

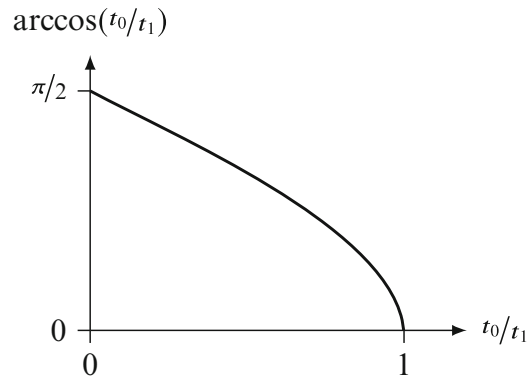

paths of the Brownian motion cross the abscissa in an interval of two consecutive points in time $\left(t_{0}, t_{1}\right)$ ? With reference to the Wiener measure discussed in Sect. 6.2 we have to determine the measure ${ }^{11}$

$$
\mu\left(\left\{f: \exists t \in\left(t_{0}, t_{1}\right) \quad f(t)=0\right\}\right) .
$$

In words: how large is the Wiener measure for all functions of the Brownian motion taking the value zero at least once in the interval $\left(t_{0}, t_{1}\right)$ ? We will only provide an answer to this question without proving it. ${ }^{12}$ Interestingly, the probability depends only on the quotient $\frac{t_{0}}{t_{1}}$. The following applies

$$
\mu\left(\left\{f: \exists t \in\left(t_{0}, t_{1}\right) \quad f(t)=0\right\}\right)=\frac{2}{\pi} \arccos \left(\frac{t_{0}}{t_{1}}\right) .
$$

The arccos function (arc cosine function) is most likely not well known to nonmathematicians. For this reason its shape is illustrated in Fig. 6.4.

What characteristics does the arccos function have if both $t_{0}$ and $t_{1}$ tend to zero? Although the quotient $\frac{t_{0}}{t_{1}}$ can take on any value, one can easily think of examples with the quotient being very small. Consider the following intervals: $\left(t_{0}, t_{1}\right)=\left(1 / n^{2}, 1 / n\right)$ with $n$ being $2,3,4, \ldots$ With increasing $n$ these intervals are getting closer and closer to zero. Since $t_{0} / t_{1}=1 / n \rightarrow 0$, the probability of a zerocrossing of the Brownian motion increases and finally approaches 1 . Thus, every single Brownian path has an infinite number of zeros in the neighborhood of its origin. Such behavior of a function is very bizarre.

Boundlessness We will now consider another interesting characteristic of the Brownian motion which is also frequently omitted in economic textbooks. Plots of Brownian paths displayed in such publications usually resemble Fig. 6.5.

Let us concentrate on a very small interval on the time axis $[t, t+\varepsilon]$ and ask the following question: what is the probability that the paths of a Brownian motion are bounded in this interval? In addressing this question we will initially focus on the upper bound. How large is the probability that all Brownian paths will not exceed an

\footnotetext{
${ }^{11}$ The symbol $\exists t$ means that "there exists a $t$ where ... applies."

${ }^{12}$ A proof can be found in Klebaner (1998, p. 76).
} 


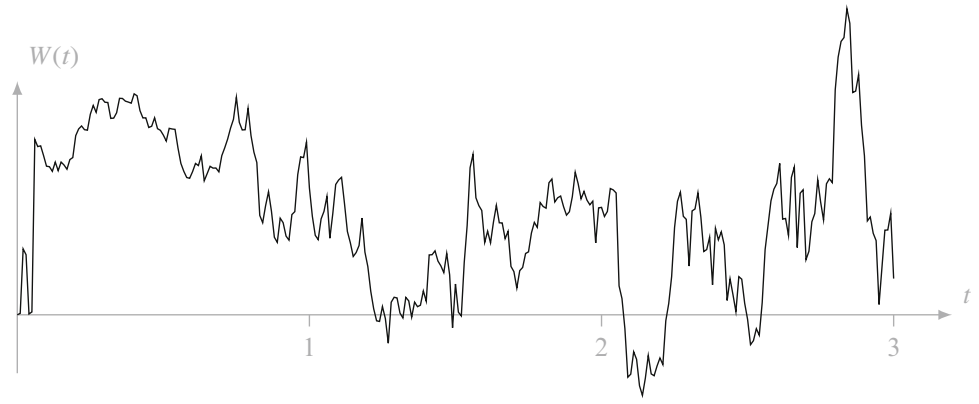

Fig. 6.5 Typical textbook image of a Brownian path

upper bound in the very small interval? More precisely: what probability measure is to be assigned to the set of all paths that will not exceed an arbitrarily high upper bound $K$ in the time interval $[t, t+\varepsilon]$ ? In the following we will show that the answer to this question is zero. If one tries to explain this result intuitively one could state: "In every small interval practically all paths are unbounded."

This assertion cannot be derived from Fig. 6.5 since the path is clearly restricted everywhere. In order to resolve this (apparent) contradiction we have to realize once again what a Brownian motion is: it is not a single path as shown in Fig. 6.5 with limited fluctuations. Rather, we are dealing with an infinite number of paths that fulfill a given property (however defined) with a certain probability. Concerning the property of unboundedness our assertion can now be stated more precisely: if we consider in the interval $[t, t+\varepsilon]$ the set of all paths having upper bounds simultaneously, ${ }^{13}$ this set has measure zero. Paths with upper bounds are therefore unlikely. They hardly ever happen.

Since proving our assertion is exceedingly involved, we will refrain from presenting a formal proof; rather we will try to substantiate the assertion by using appealing arguments that can be found in the literature on Brownian motions. Let us first remember that the Brownian motion is the set of all continuous functions $C[0, \infty)$. Continuous functions have a maximum in closed intervals; however, the issue of how this maximum is distributed remains open. A clear answer can be found in the theory of Brownian motion. For this purpose we define $K$ as the upper bound and consider the set of all paths $f(s)$ that remain below this limit in the interval $[t, t+\varepsilon]$. This set is described as

$$
\left\{f: \max _{t \leq s \leq t+\varepsilon} f(s) \leq K\right\} .
$$

\footnotetext{
${ }^{13}$ Of course the path shown in Fig. 6.5 belongs to this set.
} 
Determining the number of paths contained in this set translates to the mathematical problem of deriving the measure $\mu$ of this set which is given by ${ }^{14}$

$$
\mu\left(\left\{f: \max _{t \leq s \leq t+\varepsilon} f(s) \leq K\right\}\right)=\sqrt{\frac{2}{\pi \varepsilon}} \int_{0}^{K} e^{-\frac{x^{2}}{2 \varepsilon}} d x .
$$

It is helpful to remember that for $K \rightarrow \infty$ the following holds:

$$
\int_{0}^{\infty} e^{-\frac{x^{2}}{2 \varepsilon}} d x=\sqrt{\frac{\pi \varepsilon}{2}}
$$

For $K<\infty$, however, it follows immediately

$$
\sqrt{\frac{2}{\pi \varepsilon}} \int_{0}^{K} e^{-\frac{x^{2}}{2 \varepsilon}} d x<1
$$

Expression (6.20) implies: regardless how large $K$ is, we will never arrive at a situation where all paths of a Brownian motion fall below $K$ with probability 1 . Incidentally, this result applies to every small time interval $[t, t+\varepsilon]$ since all our statements are independent of the actual value of $\varepsilon$. Thus, it can be stated that the Brownian motion is unbounded even in the smallest interval.

To this point we only dealt with the upper bounds of Brownian paths. Using the same logic, one can also show that no lower bound exists for an infinite number of paths.

The above possibly confusing result is due to the fact that we have insisted on including all Brownian paths. However, if we consider also the probabilities of the Brownian paths we obtain results which are far less irritating. To this end we set a limit of $K$ for the upper bound and $-K$ for the lower bound. Based on our previous considerations we know that not all paths can meet finite bounds in any small interval. We are interested in finding upper and lower bounds such that only $x \%$ of all paths will fall within these bounds, however small the interval.

Figure 6.6 shows different funnels each labeled with respective probability levels. Determining these funnels or bounds for each level of probability can be accomplished by applying sophisticated mathematics; however, we restrain from presenting the details. ${ }^{15}$

A probability of $x \%$ indicates that the paths which run within the bounds constitute $x \%$ of all paths of a Brownian motion. The funnels widen with increasing probability. Of course for $x=100 \%$ the funnel is boundless.

\footnotetext{
${ }^{14}$ The distribution of the maximum can be found for example in Karatzas and Shreve (1991, p. 96).

${ }^{15}$ See Karatzas and Shreve (1991, p. 96).
} 
Fig. 6.6 The figure shows for several probabilities $x \%$ those barriers within which a total of $x \%$ of all paths of a Brownian motion run

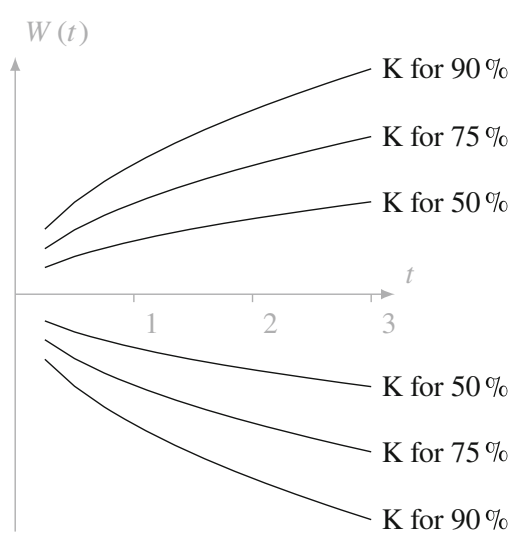

Non-monotonicity In economic textbooks Brownian paths are usually constructed by the approximation ${ }^{16}$

$$
\Delta W=\varepsilon \sqrt{\Delta t}
$$

The $\Delta W$ represents a change of a Brownian path that takes place after a (short but finite) time period of $\Delta t$ and with $\varepsilon$ being a standard normally distributed random number. ${ }^{17}$ Returning to Fig. 6.5 we see an example of a corresponding path at fixed points in time $\Delta t, 2 \Delta t, 3 \Delta t \ldots$ The path is created by linearly connecting the values approximated by Eq. (6.21). Such a piecewise linear function is of course monotonously increasing or decreasing in any time interval $\Delta t$.

However, the property of monotonicity is lost once we are abandoning the approximation approach and return to the Brownian motion. Instead of analyzing a single path one must consider the set of infinitely many paths having the property of being monotonously rising or falling in any infinitely small time interval, i.e., $\Delta t \rightarrow$ 0 . It can be shown that the measure of this set is zero. ${ }^{18}$ Monotonously growing or decreasing paths in arbitrary time intervals are therefore entirely unlikely, even if a single path may have monotonous sections.

\footnotetext{
${ }^{16}$ See for example Hull (2015, p. $\left.304 \mathrm{ff}\right)$.

${ }^{17}$ See also Eq. (1.4) on page 5.

${ }^{18}$ See for example Klebaner (2005, p. 64).
} 
Open Access This chapter is licensed under the terms of the Creative Commons Attribution 4.0 International License (http://creativecommons.org/licenses/by/4.0/), which permits use, sharing, adaptation, distribution and reproduction in any medium or format, as long as you give appropriate credit to the original author(s) and the source, provide a link to the Creative Commons licence and indicate if changes were made.

The images or other third party material in this chapter are included in the chapter's Creative Commons licence, unless indicated otherwise in a credit line to the material. If material is not included in the chapter's Creative Commons licence and your intended use is not permitted by statutory regulation or exceeds the permitted use, you will need to obtain permission directly from the copyright holder. 